\title{
Special issue on integrated and hybrid intelligent systems in product design and development
}

\author{
Xuan F. Zha \\ National Institute of Standards and Technology Gaithersburg, MD 20899, USA \\ E-mail: zha@cme.nist.gov
}

With ever increasing complexity of products and customer demands, companies are adopting new strategies to meet the changing technological requirements, shorter product life cycles, and globalization of manufacturing operations. Product design and development are getting more sophisticated procedures/processes involved and require designers and engineers possessing different expertise, knowledge and experience to work together $[1,2]$. To address these challenges, techniques based on artificial intelligence (AI) are increasingly being used to improve effectiveness and efficiency in the product design and development life cycle. Intelligent systems can be beneficially applied to many aspects of design and also design-related tasks, for example, identifying customer demands and requirements, design and planning, production, delivery, marketing and customer services, etc. Individual intelligent paradigms (such as fuzzy logic, neural network, genetic algorithm, case-based reasoning, and especially expert systems) have been applied to specific stages of the design process (especially expert systems). However, increasingly, hybrid solutions, that integrate multiple individual intelligent techniques, are required to solve complex design problems. The integrated and hybrid intelligent environment can provide various information and knowledge for supporting rapid and intelligent decision-making throughout the entire design and development process. This is in line with the evolutionary trends of product design and development process, from the traditional CAD systems into the knowledge- based engineering and integrated intelligent design systems through a combination of concurrent engineering, collaborative engineering and integrated and hybrid AI techniques. In recent years, with the advancement of $\mathrm{AI}$ and intelligent systems techniques, integrated and hybrid intelligent systems are gaining better acceptance in product design and development. The driving force behind this is that integrated and hybrid intelligence and distributed $3 \mathrm{C}$ (collaboration, cooperation, and coordination) allow the capture human knowledge and the application of it so as to achieve high quality designs/products. Further motivation arises from steady advances in individual and hybrid intelligent-systems techniques, and the widespread availability of computing resources and communications capability through intranets and the World Wide Web [1].

This special issue aims to collect relevant original works in the application of emerging integrated and hybrid intelligent systems techniques to the design of products, processes and systems in product development. The goal is to take a snapshot of the progress in the research into the support for product design and development and to disseminate how recent developments in integrated, knowledge-intensive and computational AI techniques can improve and enhance such support. The selected papers provide an integrated, holistic perspective on this complex set of challenges and provide rigorous research results. The focus of this special issue is on the integrated and hybrid intelligent methodologies, frameworks and systems for support- 
ing product design and development activities. The subject pushes the boundaries of the traditional topic of product design and development into new areas.

In [3], Yip et al. propose a methodology that involves the combined use of CAE and artificial intelligence/knowledge intensive approach to bridge the gap for the full deployment of a computer aided/virtual design alternative. The proposed model is illustrated with a case study that is concerned with the design of a plastic toaster cover with respect to a heat test. In [4], Huang et al. propose an agent-based Web services architecture applied to augment manufacturability. Not only the agent-based Web services architecture makes system interoperation feasible, but also increases the efficiency of the distributed collaboration. In [5], Manns and Tönshoff propose a neuro-fuzzy based approximation approach to forecast material-flow behavior in the offer phase. Within this approach, a neuro-fuzzy system maps material-flow simulation input parameters on performance measures. It is trained with results of multiple simulations using a model of the production line. Wu and Blackhurst [6] present a modeling methodology to manage the synthesis of a supply chain by linking hierarchical levels of the system and to model and analyze disruptions in the integrated supply chain. Kulvatunyou et al. [7] demonstrates a promising approach to integration and interoperation between a design house and a manufacturer that may or may not have prior relationship by developing semantic web services for business and engineering transactions. Ho and Ji [8] present a hybrid genetic algorithm to optimize the sequence of component placements for a chip shooter machine. A new robust paradigm focusing on e-intelligence for integrated product design, manufacturing, and service has become a new benchmark strategy for manufacturing companies to compete in the twenty-first century. In [9], Liu et al. present a research effort on the development of an Internet server controller based intelligent maintenance system for products. They also discuss how to develop products and manufacturing systems using internet-based intelligent technologies and ensure the product quality, coordinate the activities, reduce costs and change maintenance practice from breakdown reaction to breakdown prevention. In [10], Liu et al. apply feature selection technique to the data obtained from vibration signals. Artificial neural networks and soft computing are used for nonlinear pattern recognition. They make attempts to distinguish between normal and defective bearings. Furthermore, classification of roller bearing conditions into six different categories is conducted for the diag- nostic purpose. In [11], Liu and Oh developed, a PCbased Expert System, EASYMEMS, which contains domain specific knowledge of microelectromechanical systems (MEMS) as well as the reasoning process of a human expert. The computerized consultant system can be used for thorough static and dynamic analysis in MEMS design and manufacturing.

By addressing both theoretical and applicationoriented problems in this special issue. I hope that readers will find the papers stimulating and will understand some of the research challenges and future research directions. I wish to thank the authors for their contributions to this special issue and the reviewers for their thorough reviews.

\section{References}

[1] X.F. Zha, Artificial Intelligence and Integrated Intelligent Systems for Product Design and Development, in: Intelligent Knowledge-based Systems: Business and Technology in New Millennium, (Vol. IV), C.T. Leondes, ed., Chapter 1, Kluwer Academic Publishers, USA, 2004.

[2] M. Rao, Q. Wang and J.Z. Cha, Integrated Distributed Intelligent Systems in Manufacturing, Chapman and Hall, 1993.

[3] D.C.Y. Yip, M.C. Law, K.P. Cheng, K.H. Lau and S. Barnes, Knowledge intensive CAD in product design validation, International Journal of Knowledge-based and Intelligent Engineering Systems 9(2) (2005), 45-61.

[4] C.C. Huang, T.L. Tseng and H.S. Chang, An agent-based Web services solution to collaborative product design, International Journal of Knowledge-based and Intelligent Engineering Systems 9(2) (2005), 63-79.

[5] M. Manns and H.K. Tönshoff, Applicability of neuro-fuzzy function approximation in material-flow forecasting, International Journal of Knowledge-based and Intelligent Engineering Systems 9(2) (2005), 81-92.

[6] T. Wu and J. Blackhurst, A modeling methodology for supply chain synthesis and disruption analysis, International Journal of Knowledge-based and Intelligent Engineering Systems 9(2) (2005), 93-105.

[7] B. Kulvatunyou, H. Cho and Y.J. Son, A semantic Web service framework to support intelligent distributed manufacturing, International Journal of Knowledge-based and Intelligent Engineering Systems 9(2) (2005), 107-127.

[8] W. Ho and P. Ji, A hybrid genetic algorithm for sequencing PCB component placement, International Journal of Knowledge-based and Intelligent Engineering Systems 9(2) (2005), 129-136.

[9] C.L. Liu, X. Zha, Y.B. Miao and J. Lee, Internet server controller based intelligent maintenance system for e-products, International Journal of Knowledge-based and Intelligent Engineering Systems 9(2) (2005), 137-148.

[10] T.I. Liu, F. Ordukhani and D. Jani, Monitoring and diagnosis of roller bearing conditions using neural networks and soft computing, International Journal of Knowledge-based and Intelligent Engineering Systems 9(2) (2005), 149-157.

[11] T.I. Liu and C. Oh, An artificial intelligence approach for the design and manufacturing of micro electro mechanical systems (MEMS), International Journal of Knowledge-based and Intelligent Engineering Systems 9(2) (2005), 159-171. 